\title{
Clinical Study on Postpartum Urinary Retention with Compound Chinese Medicine
}

\author{
Wang Huaying and Zhang Yuemei* \\ Department of Obstetrics, Haidian District Maternity and Child Health Hospital, China
}

*Corresponding author: Zhang Yuemei, Department of Obstetrics, Haidian District Maternity and Child Health Hospital, China, E-mail: wanghuaying6688@163.com

Received: 19 Oct, 2018 | Accepted: 26 Nov, 2018 | Published: 30 Nov, 2018

Citation: Wang H, Zhang Y (2018) Clinical Study on Postpartum Urinary Retention with Compound Chinese Medicine. J Surg Open Access 4(4): dx.doi.org/10.16966/2470-0991.175

Copyright: (c) 2018 Wang $\mathrm{H}$, et al. This is an open-access article distributed under the terms of the Creative Commons Attribution License, which permits unrestricted use, distribution, and reproduction in any medium, provided the original author and source are credited.

\section{Introduction}

Postpartum urinary retention is a common complication in obstetrics; its incidence in spontaneous labor is on average $14 \%$ [1], while the incidence in midwifery is as high as $26 \%$ to $38 \%$ [2]. If the treatment is not timely, postpartum urinary retention will affect the contraction of the uterus and aggravate postpartum hemorrhage, affect the restoration of postpartum reproductive organs, can also lead to urinary tract infection, and bring physical and mental pain to the puerperal [3].

Clinical commonly used methods to resolve postpartum urinary retention include mental encouragement, warm water washing vulva or listening to the sound of running water to increase urinary intention, heating the lower abdominal or the sacral tail, massage, etc [4]. If the above methods are not effective, acupuncture therapy, the use of neostigmine and catheterization or indwelling catheterization should be adopted. Postpartum urinary retention, bladder extremely plentiful, they are dangerous when acupuncture the lower abdominal. By inhibiting the activity of cholinesterase, neostigmine can exert a cholinomimetic effect. In addition, it can directly excite the nicotinoid receptor (N2 receptor) on the motor end-plate of skeletal muscle and increase the contraction of bladder smooth muscle, but the effect is not very satisfactory. An indwelling catheter is one of the methods for treatment of urinary retention. However, in clinical application, the improper indwelling method will lead to bladder paralysis. The repeated indwelling catheter will increase the chance of urinary system infection and cause pain to patients. But Traditional Chinese Medicine (TCM) apply the umbilicus is simple, safe and worth popularizing. Shenque is located in the middle of the umbilicus, belonging to Ren meridian, which is reinforcting Yang from Yin, and according to the therapeutic principle of the traditional Chinese medicine, "treating excess with purgation, treating deficiency with tonification", fresh Ginger, natto and even the root of the green onion, external application of Shenque acupoint, pressure package to apply heat, the have Wentong, cold, turn the qi into water, so that the cold can be dispersed, lower energizer can temperature, bladder qi can put into effect, then can urinate by himself.

\section{Pathogenesis}

The main reason of postpartum urinary retention is mainly the second prolonged labor and fetal oversize, dystocia, which lead to the bladder and urethra should bear excessive pressure, which results in the bladder mucosa edema, congestion, reducing muscle tension and sensitivity of intravesical pressure drop, perineum incision pain can't urinate, lead to residual urine increase $[1,3]$. Postpartum fatigue, mental tension, afraid of pain and other conditions, so that the puerperal cannot urinate by themselves, leading to urinary retention [1]. Postpartum urinary retention is one of the important causes of postpartum hemorrhage and postpartum urinary tract infection and affects the secretion of puerperal milk, which is the focus of postpartum care.

\section{The mechanism of action of compound traditional Chinese medicine applied to Shenque acupoint}

Modern medicines consider that umbilical epidermal cutin is the thinnest, barrier function is weak, sensitivity is high, and permeability is good. Around the umbilical region, there have arteriae epigastrica superior, venae epigastrica superior, arteria epigastrica inferior venae, venae epigastrica inferior, and rich in capillaries, which has a rich capillary blood supply. The external application of drugs here is conducive to the direct absorption of drugs. This prescription to Yiqi helps in the main, blood circulation and water is auxiliary. Astragalus nourishes the qi and lifts the Yang, benefits the water to reduce the swelling, has been proved to have diuretic detumescence, antibacterial, antiviral and other functions [5]. It is helpful to restore the bladder smooth muscle and urethral sphincter tension, relieve the normal contraction and paralysis and is effective in treating postpartum urinary retention. Poria cocos inducing diuresis to alleviate edema, infiltration and strengthening the spleen, heart, and kidney meridian. The taste of poria cocos is sweet and light, sweet can mend weakness, and the diluted taste can cause seepage. It cannot only eliminate pathogenic, but also strengthen body resistance, alleviate water retention, but non-damaging the positive gas. The function of Motherwort is to invigorate the circulation of blood, inducing diuresis to alleviate edema, can enhance the contraction of smooth 
muscle by enhancing the action of $\mathrm{M}$ receptor and $\mathrm{H}$ receptor, and can improve circulation and facilitate transdermal absorption of drugs. Ginger is warm through tongluo, green onion xinwen tong Yang, enhanced lower energizer qi hua, salt belong to the kidney channel and conducive to urination. The application can treat skin diseases or systemic diseases. Compared with other dosage forms, it is safer, less toxic and side effects, effective, stable, patient painless and adaptable. Besides, it has the advantage of simple operation and saving medicine. Therefore, it can be widely used in the clinic.

\section{Clinical application of compound Chinese medicine application}

Postpartum Urinary Retention (PUR) is the common complication. For Postpartum Urinary Retention, the patients can be treated with compound Chinese medicine effectively. Prepare $15 \mathrm{~g}$ of ginger, $15 \mathrm{~g}$ of green onion, $2 \mathrm{~g}$ of thin salt, radix astragali powder $5 \mathrm{~g}$, poria cocos powder 5g, motherwort powder 5g. Clean the green onion and mash it in the mortar. Transfer salt, astragalus, poria cocos, and motherwort into a paste. Prepare all the materials to the side of the patient's bed and explain. Take the supine position, expose the navel, and to keep warm. Before dressing, use $0.9 \%$ saline cotton balls to clean the local skin. Then apply the Chinese medicine paste to the umbilical region by a tongue depressor, fix with gauze or application and press with a hot water bottle for 30 minutes. Before and after treatment should pay attention to local skin and surrounding whether fester, infection or injury. Avoid using exceptions if they occur. Use with caution if you have skin allergies. You can take the scallion section first and smear it gently on the inside of the forearm of the patient. After observing the response of the skin, if any allergic phenomena such as red rash, pruritus, and blisters occur, you should suspend the use and deal with the symptoms. After treatment, use warm water to clean the skin and observe if there is any skin redness.

\section{Questions and Prospects}

Compound Chinese medicine application is a method to apply Chinese herbal medicine to selected acupoints and treat diseases through the combined action of drugs, acupoints, and meridians. In recent years, due to the features of the simple application, low price and remarkable curative effect, the composite application of traditional Chinese medicine has been increasingly favored by a large number of patients and has a broad prospect of clinical development. At present, objective evaluation indexes should be explored, basic research should be carried out, to solve the use of patients with skin injury or allergy tendency, so as to make it a safe and effective and easy to use traditional Chinese medicine for external treatment.

\section{References}

1. Mulder FE, Oude Rengerink K, van der Post JA, Hakvoort RA, Roovers JP (2016) Delivery-related risk factors for covert postpartum urinary retention after vaginal delivery. Int Urogynecol J 27: 55-60.

2. Bouhours AC, Bigot P, Orsat M, Hoarau N, Descamps P, et al. (2011) Postpartum urinary retention. Prog Urol 21: 11-17.

3. Wilson BL, Passante T, Rauschenbach D, Yang R, Wong B (2015) Bladder Management with Epidural Anesthesia during Labor: A Randomized Controlled Trial. MCN Am J Matern Child Nurs 40: 234242.

4. Cavkaytar S, Kokanal MK, Baylas A, Topçu HO, Laleli B, et al. (2014) Postpartum urinary retention after vaginal delivery: Assessment of risk factors in a case-control study. J Turk Ger Gynecol Assoc 15: 140143.

5. Wang S, Zhang C, Yang G, Yang Y (2014) Biological properties of 6-gingerol: a brief review. Nat Prod Commun 9: 1027-1030. 